\title{
Blockchain technology for digitalization of the pharmaceutical sector of the Russian Federation
}

\author{
Ahad Qodirov* \\ Saint Petersburg State University, University embankment, 7/9, 199034 Saint-Petersburg, Russia
}

\begin{abstract}
One of the main priorities of the modern healthcare system is the transportation of pharmaceutical products. The drug supply chain consists of many participants. These are drug manufacturers, intermediary organizations, and end users. The biggest problems associated with this transportation are temperature monitoring and the quality of the offered products. This is because many drugs and vaccines remain viable within a certain temperature range. As soon as the temperature regime during transportation is violated, the quality of the medicine decreases sharply. In this article, the author proposes the use of Blockchain technology to solve these problems and focuses on improving the transportation process. In his opinion, a decentralized system based on Blockchain technology is the best technological solution. In addition, the author is developing a concept for the application of Blockchain technologies to create a functioning medical ecosystem.
\end{abstract}

\section{Introduction}

Today, the transportation of pharmaceuticals (drugs, vaccines, consumables) from an industrial manufacturer to a retail consumer (patient) is carried out according to a strict, predetermined supply chain. The most important task of this department is safe transportation.

More and more counterfeit drugs are appearing in the healthcare sector. One in ten medical products is counterfeit in most developing countries. These medicines contain the wrong medical ingredient or do not contain an active ingredient.

\section{The relevance of the technological solution}

Today, in connection with the COVID-19 pandemic, more and more attention are focused on the transportation of medicines. An investigation by the British Broadcasting Channel (BBC) News uncovered the sale of counterfeit drugs in Africa. The World Health Organization (WHO) has stated that taking these drugs can have "serious side effects" [1].

Another key problem is that many pharmaceuticals are only effective over a certain temperature range. If the transport temperature falls outside the tolerance range (too high or too low), these drugs become ineffective and therefore do not work as expected.

The ability to verify the temperature conditions and authenticity of medicines will give healthcare professionals, pharmacies, and patients a tool with which they can clearly determine the authenticity and effectiveness of the purchased medicine. When a patient or

\footnotetext{
${ }^{*}$ Corresponding author: ahadkadirov@gmail.com
} 
pharmacy (as well as other participants in the medical field) selects a suitable medicine, they will be able to verify the authenticity of the product (by tracing the supply chain) and its quality (by checking whether any of the beneficial ingredients are sensitive to the temperature conditions of storage and transportation) [2].

Patients, pharmacies, hospitals - they all rely on supply chains of logistics companies to deliver thermosensitive drugs (vaccines or insulin). When transporting these medicines over thousands of kilometers, specially designed smart shipping boxes are required that maintain a pre-calibrated temperature regime and captures all changes using a preprogrammed sensor. This drug transportation system is called a cold chain.

The essential elements of the cold chain are the transportation equipment itself for the transportation of drugs and equipment for temperature control (as well as for recording all temperature changes during transportation). In this regard, the cold chain of drug transportation requires complete trust between all parties to the transaction. Therefore, according to the author, the introduction of Blockchain technology into the transportation chain of pharmaceutical products has several advantages and benefits.

This is since, firstly, the blockchain technology can store all the sensor data that was recorded at the time of direct transportation, and secondly, because these readings cannot be changed in the system by any of the participants in the relationship. Thus, the use of Blockchain technology will build trust in the digital health logistics ecosystem. All the necessary information about how the medicinal product was transported (from the manufacturer to the end consumer) will be available to each member of the decentralized association (through the personal account of the verified user).

The smart box sensor, during transportation, can collect temperature data in a timely manner and transmit it to the Blockchain network, and so the information will already be reliably stored and available for verification and review [3 - 8].

\section{Review of existing solutions}

Initially, the author deals with reviews of existing technological solutions that are on the world market. This review will identify the strengths and weaknesses of distributed ledgers for tracking pharmaceutical products.

Blockchain technologies, due to their decentralization and since the stored information cannot be falsified, is most suitable for the transportation of medicines. Professor Sidra Malik of the University of New Wales has proposed a four-tiered structure for tracing the origin of pharmaceutical products known as TrustChain [9].

TrustChain is a blockchain application that has been developed to address trust issues that arise during the transportation of pharmaceutical products. TrustChain uses a blockchain consortium that monitors interactions between all participants in the supply chain at every stage. In addition to this, it also dynamically assigns scoring scores to participants, analyzing their activities (implementation of certain smart contracts). TrustChain system works as follows:

- Based on observations that arise during transportation, the considered Blockchain technology evaluates the quality of products and the reliability of logistics organizations.

- After assessing the reliability of the organization, the system exposes reputation (scoring) ratings to each participant in the supply chain. In addition, TrustChain evaluates the specific pharmaceutical product that has been transported.

- To assess the reliability of the logistics organization and the quality of the goods, TrustChain uses smart contracts to automatically, secure, efficiently, and transparently calculate points.

- The overhead costs of maintaining the Blockchain structure (in terms of throughput and latency) are minimal compared to other supply chain models. 
Professor at the University of Trento Miguel P. Caro presented another Blockchain development for drug transportation - AgriBlockIoT. The proposed model integrates distributed ledger technology to create verifiable, transparent, and immutable records that are used to track the supply chain. This model used two different Blockchains - Ethereum and Hyperledger [10].

Professor Ruchi Vyas from Dr. D. Patel University presented a comprehensive analysis of the pharmaceutical supply chain. She analyzed all the benefits of Blockchain technology for the supply chain of pharmaceuticals, and also paid attention to the problems of temperature regimes [11].

\section{Materials and methods}

The logistics chain is an extensive network of independent nodes, which are represented by various participants. These participants include pharmaceutical manufacturers, medical intermediaries, technology solutions, insurance organizations, end users, and regulatory authorities. The supply chain starts from the supplier of pharmaceutical raw materials and ends with the consumer of drugs. Consumers can be both medical structures (hospitals, pharmacies, clinics) and individuals (patients). The supply chain is designed for transportation (as well as storage during transportation) of manufactured medicines from the point of production to the point of destination. The pharmaceutical supply chain includes the following important elements:

- Manufacturer of pharmaceutical products. The manufacturer receives actual orders from wholesale distributors and dispatches finished pharmaceutical products.

- Distributor of pharmaceutical products. A distributor is a wholesaler who distributes (purchased from a supplier) pharmaceutical products. Distribution takes place along predefined logical chains (under contracts with pharmacies and hospitals).

- Pharmacy institutions. Pharmacies and hospitals purchase pharmaceuticals from distributors. Medicines are sold at retail prices to patients and other customers.

An important aspect is that some pharmaceutical products require a specific, welldefined, temperature range. This temperature range must be maintained regardless of whether drugs are stored at the distributor's warehouse or are in transit. In this regard, a logistic supply chain that requires temperature control is called cold transport. Cold transport consists of several aspects, described below:

Cold storage of the medicinal product. Cold storage requires a specific cooling system. This system is used to maintain a constant required temperature. In this regard, logistics companies specially equip warehouses with refrigerating chambers and temperature storage chambers.

Cold transport of the medicinal product. Logistics companies supply their transport vehicles with special refrigerators and isothermal containers that maintain a constant temperature during the transport cycle.

\section{Problems of the question under study}

Today, the logistics system for pharmaceutical products in the Russian Federation has several problems. These problems include the following points:

- Counterfeiting of medicinal products. During the transportation process, drugs are transferred to the end user without the participation of the buyer himself. In this regard, original drugs can be substituted at any level of the supply chain. Of course, some pharmaceutical companies use holographic technology to help reduce the risk of tampering. 
But, on the one hand, such types of pharmacological protection are expensive, and on the other hand, such technological solutions can also be hacked.

- Temperature violations. When transporting thermosensitive pharmaceuticals (insulin, immunobiologicals, vaccines), it is important to monitor the temperature variation. Full temperature monitoring throughout the entire supply chain is the most important aspect of product quality. Temperature regimes are now monitored using transport sensors that send data to a single storage center. This is a "bottleneck" in the supervision system since a single center is centralized. Hence, it can be hacked, and data compromised.

- Secondary resale of medicinal products. Now, in the Russian Federation, the online checkout system is widely used, which allows to reduce the level of secondary resale of drugs. However, low-quality pharmaceutical companies have found methods to "work around" this system. First, pharmacies set up online cash registers so that they do not send all punched checks to the tax office, but only some of them. Secondly, they are engaged in artificial retail overpricing [12].

In this regard, for large pharmaceutical companies, effective supply chain management is associated with high complexity. But the creation of a functioning and safe mechanism that will allow tracking the quality of products (originality-falsification) and temperature regime will create conditions for the development of the pharmacological market and increase the profitability of its participants.

According to the statistical agency BIR Research, pharmaceutical companies will be the most visible beneficiaries of the implementation of Blockchain technology in the logistics structure. Every year, according to the agencies, due to counterfeit drugs, this segment of the market receives less than $\$ 200$ billion. The market analytics report "Global Blockchain in Healthcare - Analysis and Forecast, 2018-2025" states that by ensuring transparency in the supply chain, the technology under consideration will reduce product counterfeiting and reduce revenue losses for pharmaceutical companies by $\$ 43$ billion per year [13].

According to the World Health Organization, the market for counterfeit medicines (pharmacological tampering) is a whopping $\$ 75$ billion in 2021. This problem is most relevant in low-income countries, where there is no production pharmacological base, and many costs go to importing suppliers [14].

As an example, the following statistics can be cited. In 2019, a five-month operation by Interpol in China and seven of its neighbors in Southeast Asia, seized more than 20 million pills with counterfeit drugs from the total circulation. In addition, in Egypt, counterfeit drugs worth two hundred and thirty million dollars have been identified and criminal networks that sell fake pharmaceutical products throughout the Middle East have been exposed. According to Sabine Kopp, a former interim secretary of IMPACT and head of the WHO anticounterfeiting program, the main problem with these counterfeiting is the unreliability of existing logistics technologies.

\section{Results and technological solutions}

According to the author, with the correct use of distributed ledgers, this technology can be an excellent solution for improving the entire supply chain, from the origin of data to its integrity. This opinion is based on the following factors.

First, the blockchain fully meets all the criteria required for DSCSA compliance. DSCSA is an international Drug Supply Chain Security Act that aims to prevent the introduction and distribution of counterfeit drugs. The initiator of this law is the United States. DSCSA criteria include primary identification of a medicinal product, full traceability during transport, inspection, detection, and response to tampering (notification of the relevant agencies), as well as the exchange of necessary information between the parties to the relationship (request-sending of licenses, permits, quality certificates, etc.) [15]. 
Secondly, blockchain is responding to digital trends in the pharmaceutical market. Today, pharmaceutical companies are actively partnering with mature technology firms in distributed ledgers to develop, implement, and manage their pharmaceutical supply chain. A clear example of this is the collaboration between pharmaceutical giant GlaxoSmithKline and blockchain company Viant. This partnership (technological result) has greatly facilitated the implementation and use of Blockchain technology used by pharmaceutical companies to track the supply chain of pharmaceutical products.

The use of Blockchain technology will minimize the listed problems. This is due to the following factors:

- due to its decentralized property and the absence of a single information storage center, blockchain will strengthen trust in the digital ecosystem.

— using a high-performance blockchain network (BDN), bloXroute server and the Raft consensus algorithm, an environment is created in which data falsification is minimized. This is since in the proposed system it is easy to identify which data on pharmacological preparations have been manipulated and which have not (from the manufacturer to pharmacies) [16].

The author proposes to use the specified distributed network (BDN, bloXroute, Raft) because the Blockchain bandwidth is a bottleneck of the technology in question. As an example, the following data can be given. The throughput of the public Ethereum blockchain (its latest version) is approximately 10-30 TPS, while the throughput of the Bitcoin blockchain is 3-7 TPS. This suggests that the bandwidth that is available for the Bitcoin and Ethereum blockchain networks is not sufficient for pharmaceutical applications where higher bandwidth is needed.

For a pharmacological supply chain implemented on a national scale, at least 1000 TPS is required for the entire technological structure. Raft Consensus Algorithm coupled with bloXroute distributed network best suits technology scalable requirements.

The proposed blockchain structure only stores supply chain events, while the actual data is stored in a distributed cloud. Data stored in the cloud is represented as identical blocks that are directly associated with a unique block number. These clouds integrate with bloXroute servers and peer-to-peer networking. The hash function of the logistic data is calculated using the Merkle tree structure. This leads to the fact that each individual block has its own Merkle root. The Merkle structural tree itself is stored in the Blockchain. All this technological structure leads to the fact that any data manipulation (falsification, duplication, hiding) becomes impossible. In this regard, this technological proposal solves the three abovementioned logistic problems (falsification of drugs, violation of the temperature regime, secondary resale). The described technological structure of a scalable blockchain network is shown in Figure 1. 


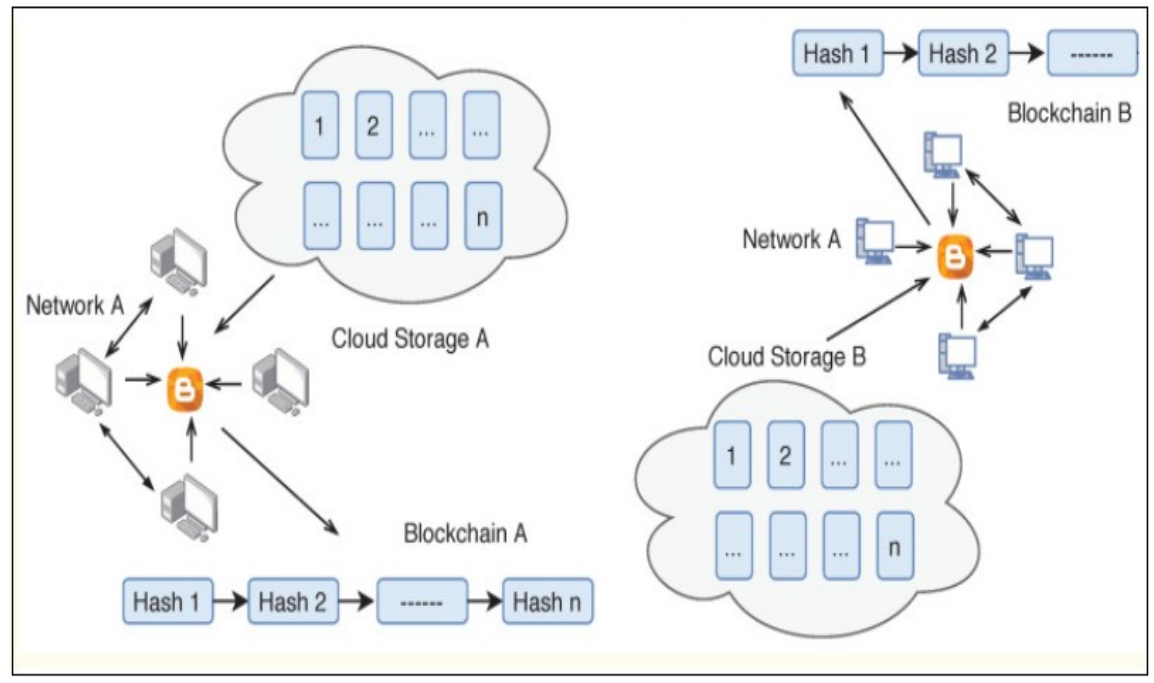

Fig. 1. Scalable and high performance blockchain distribution network (bloXroute server and Raft consensus algorithm).

Falsification of a drug becomes impossible because drug data is stored in a distributed ledger. Therefore, their change and unauthorized modifications are impossible. By building a supply chain from the drug manufacturer to the end consumer (based on Blockchain technology), the healthcare system excludes the penetration of substandard and counterfeit products.

Violation of the temperature regime (concealment of the fact of violation) also becomes impossible. This is since the data sent by the temperature sensors are sent to the decentralized network and, therefore, cannot be corrected or hidden by one of the participants in the supply chain.

Having created a single unchanging information field, the third problem is being solved - the secondary resale of drugs. According to the authors, the secondary resale and bypass of the CCP is possible because the buyer himself has been removed from the sales chain. But by creating a unified system of the buyer, the pharmacy and the supervisory authority, these problems can be solved. A schematic representation of the sales tracking chain is shown in Figure 2. 


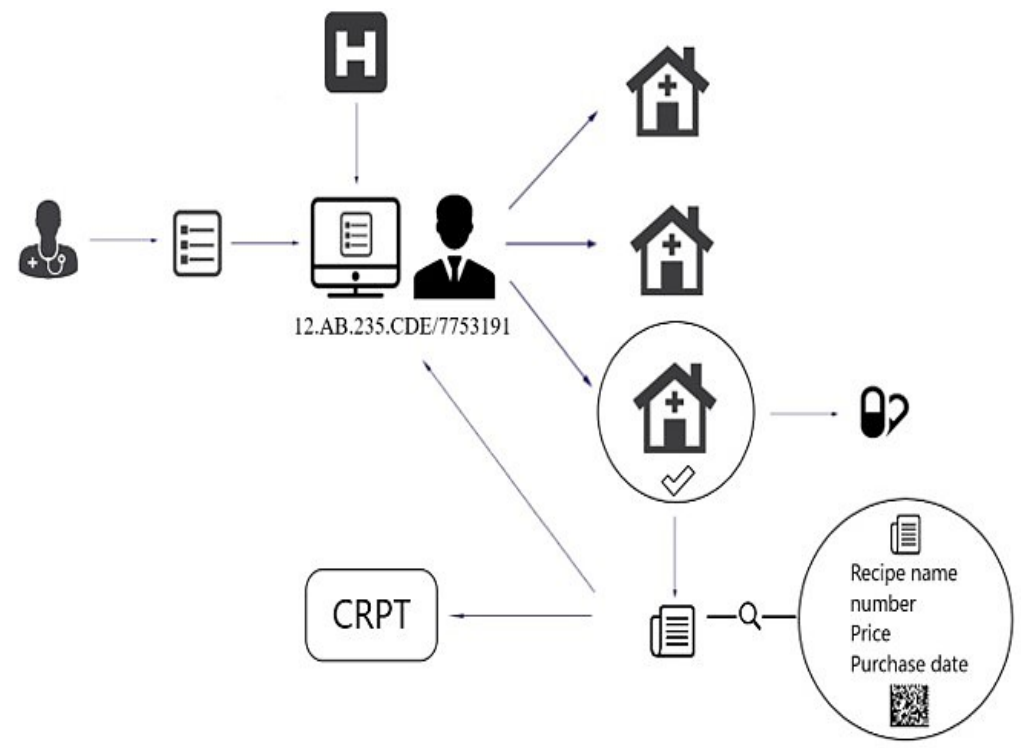

Fig. 2. A model for tracking the sale of drugs.

This system works as follows. The doctor, having assigned a course of treatment to his patient, writes him an electronic prescription (through his personal doctor's office). The recipe is displayed in the patient's personal account. Each patient and each doctor has their own personal account in the blockchain system. All personal data is hashed by the system and is protected from third-party use. The patient's personal account is tied to his SNILS. The doctor's personal account is identified by the network administrator.

After receiving the prescription, the patient goes to the pharmacy. Using his key card with a private access code, he holds it to the scanning device and shows his prescription to the pharmacy. The pharmacy issues him the prescribed drugs and sends a check (indicating the drugs received, the number of names, the total amount to be paid, etc.) to his personal account, as well as to the personal address of the supervisory authority (in this case, the Federal Tax Service, and the insurer services). This information in the personal account can be viewed by the user himself, his attending physician, insurance agent and supervisory authority. A documentary representation of the work of the technology for the implementation of pharmacological preparations is presented in Figure 3.

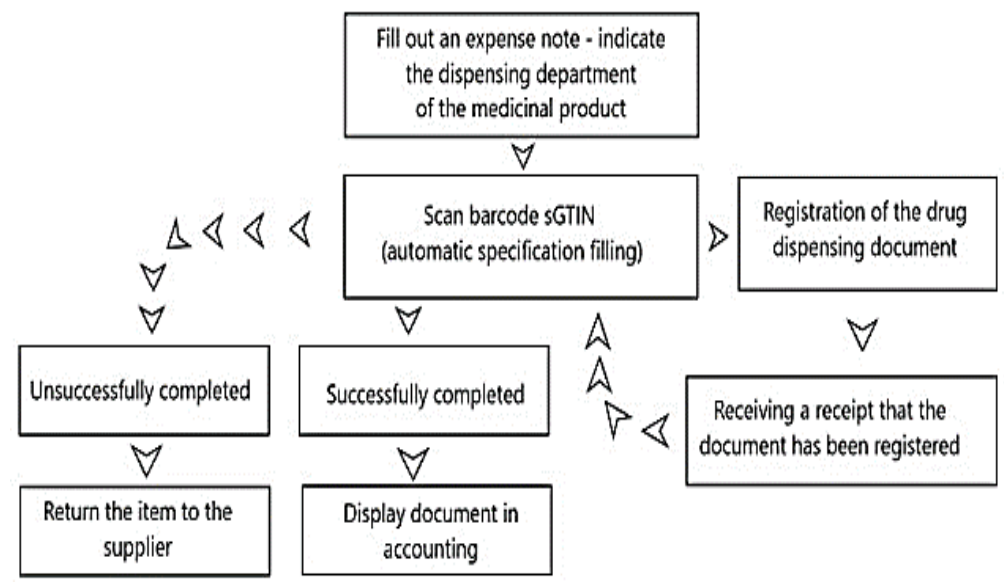


Fig. 3. Dispensing a medicinal product by a medical institution.

As a result of using Blockchain technology, the Russian logistics structure will receive a transparent system. The pharmacy will not be able to resell its products again, since it sends an information receipt to the user (and duplicates it to the supervisory and insurance authority), where the exact price is indicated. This information can be viewed by both the supervisor and the patient himself.

The creation of a unified information system based on Blockchain technology will solve the problems of the shadow market for the secondary resale of drugs, which will have a positive effect on the entire healthcare infrastructure. The world community knows examples of successful integration of Blockchain technologies into their healthcare system. A striking example of such integration is Estonia, which, during digitalization of medicine with the help of this technology, was able to raise the level of social well-being of the population by almost 15 percent in three years [17].

\section{Conclusions}

The digitalization of domestic healthcare using distributed ledgers is aimed at developing better quality medical products. It contributes to the development of the sector in question and leads to the improvement of the entire structure.

The modern Russian health care system is becoming more and more patient-oriented, the possibility of a personalized approach to his health and personal needs in ensuring the quality of services. This leads to the fact that the problems existing on the pharmacological market are beginning to be actively studied and technologically corrected. In this regard, new proposals for their solution appear.

Currently, there is a certain trend in the medical services market - this is portability and mobility, security, and high technology. Digitalization of the pharmacological sector with the help of distributed registries will not only provide comfort to patients, but also lead to a reduction in the patient's stay in inpatient treatment, which will reduce the cost of treatment. In addition, this technology can reduce the "gray" market in the pharmaceutical sector, which will lead to an increase in tax collections and an increase in competitive advantages.

Substandard and counterfeit medicines are a major problem in the pharmaceutical industry. The global market for substandard medicines is estimated at $\$ 200$ billion. Distributed registries can solve this problem, providing full information transparency at all stages of the supply of medicines. And due to its immutability, this technology minimizes the forgery of stored information (due to the fact that several parties take part in each transaction, and the information itself is decentralized).

Counterfeit medicines pose a threat not only to the health of end users, but also undermine the reputation of pharmaceutical companies. And this directly reduces the economic performance of the sector as a whole. Entering all the logistics information into a distributed ledger will help solve this problem as well. Pharmaceutical and logistics companies will be able to confirm the authenticity of the data at any time, and patients will be able to make sure that the pharmacological agent is genuine.

The analysis shows great opportunities in the development of the healthcare system using Blockchain technologies. These prospects are aimed at all market participants. And on patients who will receive better services from the medical sector from this implementation. And for private companies, which this technology will give opportunities to implement not only socially-oriented business, but also to generate large incomes. And it is also focused on the state - the Blockchain technology will reduce the costs of production and logistics. And this, in turn, will increase the overall level of economic well-being in the country. 


\section{References}

1. R. Anand, K. Niyas, S. Gupta, S. Revathy, Inventive Communication and Computational Technologies, 1223 (2020)

2. C. Sohrabi, Z. Alsafi, N. O’Neill, M. Khan, A. Kerwan, A. Al-Jabir, C. Iosifidis, R. Agha, Int. J. Surg., 76, 71 (2020)

3. A. Panarello, N. Tapas, G. Merlino, F. Longo, A. Puliafito, Blockchain and iot integration: A systematic survey (2018)

4. S. Figorilli, F. Antonucci, C. Costa, F. Pallottino, L. Raso, M. Castiglione, E. Pinci, D. Del Vecchio, G. Colle, A. R. Proto, A blockchain implementation prototype for the electronic open source traceability of wood along the whole supply chain (2018)

5. A. Dorri, S. S. Kanhere, R. Jurdak, Proceedings of the 2017 IEEE/ACM Second International Conf. on Internet-of-Things Design and Implementation (IoTDI), 173 (2017)

6. A. D. Dwivedi, Int. J. Adv. Technol., 18 (2014)

7. A. D. Dwivedi, G. Srivastava, S. Dhar, R. Singh, A decentralized privacy-preserving healthcare blockchain for IoT (2019)

8. A. D. Dwivedi, L. Malina, P. Dzurenda, G. Srivastava, Proceedings of the 42nd International Conf. on Telecommunications and Signal Processing, TSP, 135 (2019)

9. S. Malik, V. Dedeoglu, S. S. Kanhere, R. Jurdak, Proceedings of the 2019 IEEE International Conf. on Blockchain (Blockchain), 184 (2019)

10. M. P. Caro, M. S. Ali, M. Vecchio, R. Giaffreda, Proceedings of the 2018 IoT Vertical and Topical Summit on Agriculture-Tuscany (IOT Tuscany), 1 (2018)

11. D. Kapoor, R. Vyas, D. Dadarwal, Drug. Des. Int. Prop. Int. J. (2018)

12. A. V. Chernyshev, A. B. Lutsev, L. A. Gornostaeva, TSU Bulletin (2015)

13. Global Blockchain in Healthcare Market Focus on Industry Analysis and Opportunity Matrix - Analysis and Forecast, 2018-2025

14. Bulletin of the World Health Organization

15. Drug Supply Chain Security Act (DSCSA)

16. K. Uri, B. Soumya, K. Aleksandar, S. E. Gun, bloXroute: A Scalable Trustless Blockchain Distribution Network (2020)

17. Distributed ledger in healthcare: why medicine needs a blockchain 